\title{
Ramsey Method of Separated Oscillatory Fields for High-Precision Penning Trap Mass Spectrometry
}

\author{
S. George, ${ }^{1,2, *}$ S. Baruah, ${ }^{3}$ B. Blank, ${ }^{4}$ K. Blaum,,${ }^{1,2}$ M. Breitenfeldt, ${ }^{3}$ U. Hager, ${ }^{5}$ F. Herfurth, ${ }^{1}$ A. Herlert, ${ }^{6}$ A. Kellerbauer, \\ H.-J. Kluge, ${ }^{1,8}$ M. Kretzschmar, ${ }^{2}$ D. Lunney, ${ }^{9}$ R. Savreux, ${ }^{1}$ S. Schwarz, ${ }^{10}$ L. Schweikhard, ${ }^{3}$ and C. Yazidjian ${ }^{1}$ \\ ${ }^{1}$ GSI, Planckstraße 1, 64291 Darmstadt, Germany \\ ${ }^{2}$ Institut für Physik, Johannes Gutenberg-Universität, 55099 Mainz, Germany \\ ${ }^{3}$ Institut für Physik, Ernst-Moritz-Arndt-Universität, 17487 Greifswald, Germany \\ ${ }^{4}$ Centre d'Etudes Nucléaires de Bordeaux-Gradignan, 33175 Gradignan Cedex, France \\ ${ }^{5}$ Department of Physics, University of Jyväskylä, P.O. Box 35 (YFL), 40014 Jyväskylä, Finland \\ ${ }^{6}$ Physics Department, CERN, 1211 Geneva 23, Switzerland \\ ${ }^{7}$ Max-Planck Institut für Kernphysik, 69117 Heidelberg, Germany \\ ${ }^{8}$ Physikalisches Institut, Ruprecht-Karls-Universität, 69120 Heidelberg, Germany \\ ${ }^{9}$ CSNSM-IN2P3-CNRS, 91405 Orsay-Campus, France \\ ${ }^{10}$ NSCL, Michigan State University, East Lansing, Michigan 48824-1321, USA \\ (Received 7 December 2006; published 16 April 2007)
}

\begin{abstract}
Ramsey's method of separated oscillatory fields is applied to the excitation of the cyclotron motion of short-lived ions in a Penning trap to improve the precision of their measured mass values. The theoretical description of the extracted ion-cyclotron-resonance line shape is derived and its correctness demonstrated experimentally by measuring the mass of the short-lived ${ }^{38} \mathrm{Ca}$ nuclide with an uncertainty of $1.1 \times 10^{-8}$ using the Penning trap mass spectrometer ISOLTRAP at CERN. The mass of the superallowed beta emitter ${ }^{38} \mathrm{Ca}$ contributes for testing the theoretical corrections of the conserved-vector-current hypothesis of the electroweak interaction. It is shown that the Ramsey method applied to Penning trap mass measurements yields a statistical uncertainty similar to that obtained by the conventional technique but 10 times faster. Thus the technique is a new powerful tool for high-precision mass measurements.
\end{abstract}

In 1989 the Nobel prize for physics was awarded in part to N.F. Ramsey [1] in recognition of his molecular beam resonance method with spatially separated oscillatory fields [2,3]. In 1992, G. Bollen et al. [4] demonstrated the use of time-separated oscillatory fields for the excitation of the cyclotron motion of an ion confined in the Penning trap spectrometer ISOLTRAP. Along with further experiments $[5,6]$, this showed that the method could improve the precision of mass measurements with Penning trap-on the condition that a sound theoretical basis be provided to describe the shape of the ion-cyclotron resonance curve.

In this Letter, we introduce the correct theoretical description of the application of the Ramsey method to ions stored in a Penning trap. We also demonstrate its validity for the first time with a mass measurement. Comparison with the conventional excitation scheme $[7,8]$ shows that the linewidth of the resonance is reduced by almost a factor of 2 and the statistical uncertainty of the extracted resonance frequency is more than a factor of 3 smaller. We show that the Ramsey method allows a measurement with the same statistical uncertainty but 10 times more rapidly. Faster experiments are desirable in any field since they make measurements less vulnerable to systematic errors and equipment failure. In particular, measurements of short-lived species at radioactive-beam facilities [9] benefit greatly due to the low production rates and extremely limited beam time. Such is the case of ${ }^{38} \mathrm{Ca}$, measured here $\left(T_{1 / 2}=440(8) \mathrm{ms}\right)$. This nuclide is of interest for testing the conserved-vector-current (CVC) hypothesis of the standard model of particle interactions $[10,11]$ for which a particularly high precision is required $\left(10^{-8}\right)$.

The prerequisite for the successful implementation of the Ramsey method to stored ions is the detailed understanding of the observed time-of-flight cyclotron resonance curves using time-separated oscillatory fields. While here only the most important parts of the theory and its experimental confirmation is given, a detailed presentation will be published elsewhere.

In a Penning trap an ion with a charge-to-mass ratio $q / m$ is stored in a strong homogeneous magnetic field $B_{0}$ combined with a weak electrostatic quadrupole field. The mass measurement is performed via the determination of the cyclotron frequency $\nu_{c}=q B_{0} /(2 \pi m)$. For the basic theory of the ion motion in Penning traps we refer to the review article of Brown and Gabrielse [12]. Our considerations here focus on the process of conversion of the magnetron mode into the cyclotron mode under the influence of the quadrupolar rf field in the Penning trap, extending previous studies by one of us [13] of this process in a quantum mechanical framework. In this work the effective interaction of a quadrupolar field with driving frequency $\nu_{d} \approx \nu_{c}$ with a trapped ion is described in terms of the annihilation $(a)$ and creation $\left(a^{\dagger}\right)$ operators of the magnetron $(-)$ and cyclotron $(+)$ oscillators, the phase of the quadrupole field $\phi_{d}(t)=\omega_{d} t+\chi_{d}$ with $\omega_{d}=2 \pi \nu_{d}$, 
and a coupling constant $g$ that is proportional to the amplitude of the quadrupolar field:

$$
H_{1}(t)=\hbar g\left[e^{-i \phi_{d}(t)} a_{+}^{\dagger}(t) a_{-}(t)+\text { H.c. }\right],
$$

"H.c." denotes the Hermitian conjugate of the first term. Addition of this interaction to the Hamiltonian of an ion in an ideal Penning trap yields a model system for which Heisenberg's equations of motion can be solved rigorously. The interaction (1) has the important property that the total number of quanta in the magnetron and cyclotron oscillators is conserved, $N_{\text {tot }}=N_{+}(t)+N_{-}(t)=a_{+}^{\dagger}(t) a_{+}(t)+$ $a_{-}^{\dagger}(t) a_{-}(t)=2 T_{0}$. The conversion process can be studied in terms of the "Bloch vector operator" $\mathbf{T}=T_{1} \mathbf{e}_{1}+$ $T_{2} \mathbf{e}_{2}+T_{3} \mathbf{e}_{3}$, introduced in [13], with

$$
\begin{aligned}
& T_{1}(t)=\frac{1}{2}\left[a_{+}^{\dagger}(t) a_{-}(t)+a_{-}^{\dagger}(t) a_{+}(t)\right], \\
& T_{2}(t)=\frac{1}{2 i}\left[a_{+}^{\dagger}(t) a_{-}(t)-a_{-}^{\dagger}(t) a_{+}(t)\right], \\
& T_{3}(t)=\frac{1}{2}\left[a_{+}^{\dagger}(t) a_{+}(t)-a_{-}^{\dagger}(t) a_{-}(t)\right] .
\end{aligned}
$$

The components obey the same commutation rules as those of angular momenta, $\left[T_{j}, T_{k}\right]=i \epsilon_{j k l} T_{l}(j, k, l=1,2,3)$ and $T_{1}^{2}+T_{2}^{2}+T_{3}^{2}=T_{0}\left(T_{0}+1\right)$. The expectation value $\langle\mathbf{T}\rangle$ is a real three-dimensional vector of constant length that describes a precessional motion on a "Bloch sphere" during the quadrupolar excitation. The model Hamiltonian expressed in terms of $\mathbf{T}$

$$
\begin{aligned}
H(t)= & \hbar \omega_{1}\left(T_{0}+\frac{1}{2}\right)+\hbar \omega_{c} T_{3}(t) \\
& +\hbar 2 g\left[\cos \phi_{d}(t) T_{1}(t)+\sin \phi_{d}(t) T_{2}(t)\right]
\end{aligned}
$$

governs the time development of the Bloch vector operator. Compare this result to the Hamiltonian $H_{\text {mag }}$ that describes the precession of the nuclear spin $\mathbf{I}$ in magnetic resonance experiments, $\mathbf{I}=I_{1} \mathbf{e}_{1}+I_{2} \mathbf{e}_{2}+I_{3} \mathbf{e}_{3}$,

$$
H_{\mathrm{mag}}=-\vec{\mu} \mathbf{B}=-\hbar \omega_{L} I_{3}-\hbar \gamma B_{1}\left(\cos \omega t I_{1}-\sin \omega t I_{2}\right),
$$

where $\vec{\mu}=\gamma \mathbf{I}$ is the nuclear magnetic moment, $\gamma$ the gyromagnetic ratio, and $\omega_{L}$ the Larmor frequency. Both Hamiltonians govern the time development of a vector operator, thus exhibiting a dynamical similarity between nuclear magnetic resonance on the one hand and ion motion in a Penning trap with quadrupole excitation on the other hand. This structural analogy provides deeper insight as to why Ramsey's idea of using separated oscillating fields in magnetic resonance experiments can be successfully applied also to Penning trap physics.

Initially $(t=0)$ the ions are prepared in a pure magnetron mode $\left(N_{\text {tot }}=N_{-}(0)=-2\left\langle T_{3}(0)\right\rangle\right)$, with the objective to convert the magnetron motion as completely as possible into cyclotron motion and thus to bring the radial energy to its maximum. For the conventional excitation scheme with a single pulse [see Fig. 1(a)] of duration $\tau$ and constant amplitude the expectation value for the relative number of converted quanta is obtained as

$$
F_{1}\left(\omega_{R}, \tau, g\right)=\frac{N_{+}(\tau)}{N_{\text {tot }}}=\frac{4 g^{2}}{\omega_{R}^{2}} \sin ^{2}\left(\omega_{R} \tau / 2\right),
$$

where $\omega_{R}=\sqrt{(2 g)^{2}+\delta^{2}}$ is the analog of the Rabi frequency and $\delta=\omega_{d}-\omega_{c}$ the detuning of the quadrupolar field. The "conversion time" required for complete conversion exactly on resonance is $\tau_{c}=\pi /(2 g)$.

If two pulses of quadrupolar radiation, each of duration $\tau_{1}$, and separated by a waiting period $\tau_{0}$ [see Fig. 1(b)], are used for the excitation, the expectation value for the relative number of converted quanta becomes

$$
\begin{aligned}
F_{2}\left(\delta, \tau_{0}, \tau_{1}, g\right)= & \frac{16 g^{2}}{\omega_{R}^{2}} \sin ^{2}\left(\frac{\omega_{R} \tau_{1}}{2}\right)\left[\cos \frac{\delta \tau_{0}}{2} \cos \frac{\omega_{R} \tau_{1}}{2}\right. \\
& \left.-\frac{\delta}{\omega_{R}} \sin \frac{\delta \tau_{0}}{2} \sin \frac{\omega_{R} \tau_{1}}{2}\right]^{2} .
\end{aligned}
$$

In order to compare experimentally the different excitation methods the total duration of the excitation cycle was chosen equal for both, namely, $900 \mathrm{~ms}$. The calculated energy conversion ratio is shown in Fig. 2 as a function of the frequency detuning $\delta^{\prime}=\delta /(2 \pi)$ and the waiting time $\tau_{0}$. The conventional single pulse excitation appears here as the limiting case with waiting period $\tau_{0}=0$. The experimental resonance spectra for a conventional scheme as well as for a scheme with two excitation periods of $\tau_{1}=$ $100 \mathrm{~ms}$ and $\tau_{0}=700 \mathrm{~ms}$ are shown in Fig. 3. In the latter case the sidebands are very pronounced and the full width at half maximum (FWHM) is considerably reduced. The solid line represents the fit of the theoretical line shape to the data points.

The mass measurements on the short-lived nuclide ${ }^{38} \mathrm{Ca}$ using the Ramsey method were performed using the Penning trap mass spectrometer ISOLTRAP [14] installed at the online mass separator facility ISOLDE/CERN. The calcium isotope was produced by bombarding a heated

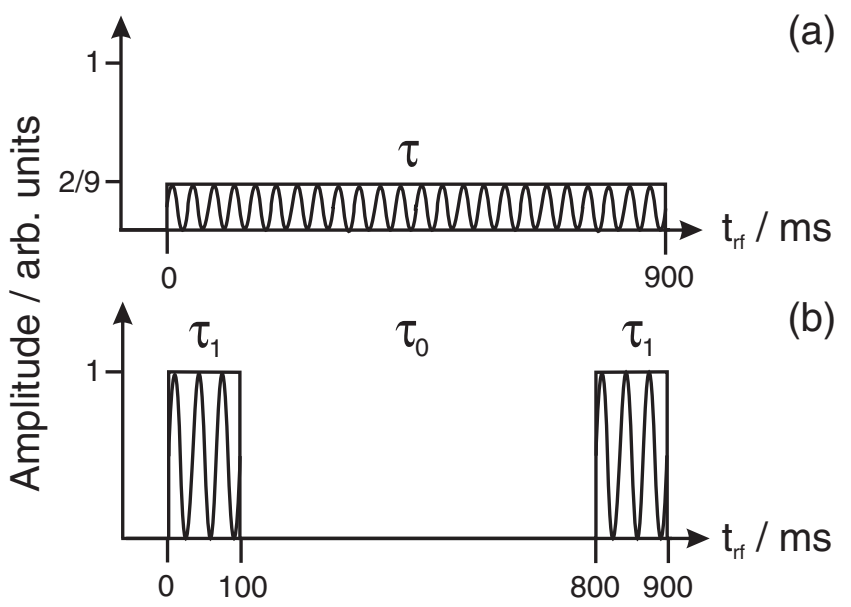

FIG. 1. (a) Conventional excitation scheme with a continuous rf pulse (here $\tau=900 \mathrm{~ms}$ ). (b) Excitation with two 100-ms Ramsey pulses $\tau_{1}$ interrupted by a $700 \mathrm{~ms}$ waiting period $\tau_{0}$. 


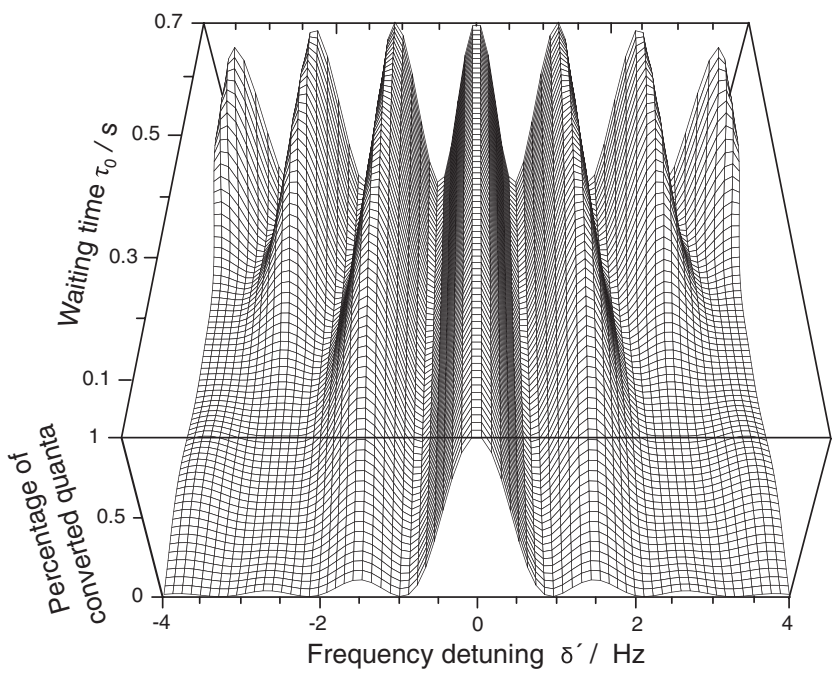

FIG. 2. Percentage of converted quanta vs the frequency detuning $\delta^{\prime}=\delta /(2 \pi)$ and vs the waiting time $\tau_{0}$ of the two-pulse Ramsey scheme of Fig. 1(b). Each pulse has a duration of $\tau_{1}=$ $\tau_{c} / 2$ to obtain complete conversion at resonance; the total duration of the excitation cycle is fixed to $900 \mathrm{~ms}$.

titanium foil target with $1.4-\mathrm{GeV}$ protons from the CERN proton-synchrotron-booster accelerator. A hot tungsten surface was used to ionize the released atoms. In order to suppress isobaric contaminations by ${ }^{38} \mathrm{~K}^{+}$ions, $\mathrm{CF}_{4}$ was added and the ions of interest were delivered to ISOLTRAP in form of the molecular sideband ${ }^{38} \mathrm{Ca}^{19} \mathrm{~F}^{+}$. Ions extracted from the source were accelerated to $30 \mathrm{keV}$, mass separated in ISOLDE's high-resolution mass separator, and injected into the first part of the ISOLTRAP apparatus, a gas-filled linear radio-frequency quadrupole ion trap for accumulation, cooling, and bunching of the ion beam [15]. From here the ions were transferred at lower energy as short bunches to a cylindrical Penning trap for further buffer-gas cooling and isobaric purification [16]. The actual mass measurement was performed in a second, hyperboloidal Penning trap [17] via the cyclotron-frequency determination.

The time-of-flight ion-cyclotron-resonance detection technique [7] relies on the coupling of the ion's orbital magnetic moment to the magnetic-field gradient after excitation of the ion motion with rf fields and axial ejection from the trap into a time-of-flight section. The sum frequency of the cyclotron mode and the magnetron mode $\nu_{c}=\nu_{+}+\nu_{-}$is probed by the measurement of the radial energy $[18,19]$. A typical time-of-flight cyclotron resonance of ${ }^{38} \mathrm{Ca}^{19} \mathrm{~F}^{+}$ions using the conventional excitation technique with one continuous rf pulse [Fig. 1(a)] is shown in Fig. 3(a). The mass of the ion of interest is obtained from a comparison of its cyclotron frequency with the one of a reference ion with well-known mass, here ${ }^{39} \mathrm{~K}^{+}$.

The uncertainty of the center frequency depends mainly on three parameters. First, it is inversely proportional to the square root of the number of recorded ions; second, it is proportional to the linewidth of the resonance; and third, it
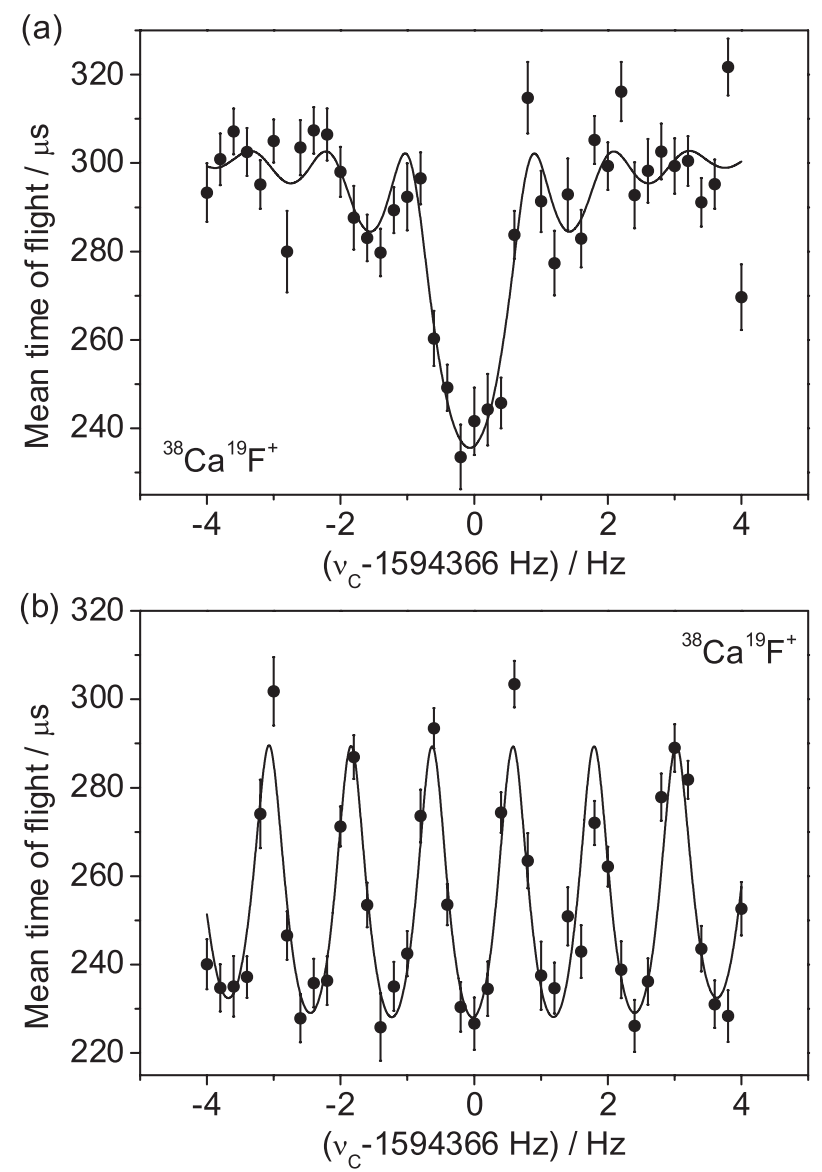

FIG. 3. Time-of-flight ion-cyclotron-resonance spectra of ${ }^{38} \mathrm{Ca}^{19} \mathrm{~F}^{+}$with the conventional quadrupolar excitation (a) and with a two-pulse Ramsey scheme with two $100 \mathrm{~ms}$ duration excitation periods interrupted by a $700 \mathrm{~ms}$ waiting period (b). The solid curves are fits of the theoretical line shapes to the data. The center frequency, where the detuning is 0 , is at $1594365.92 \mathrm{~Hz}$.

depends on the shape of the resonance and its sidebands. The stronger the sidebands are, the more precisely the center frequency can be determined. For the latter two aspects Ramsey's excitation method with separated oscillatory fields is superior to the conventional method.

Altogether eight resonances of ${ }^{38} \mathrm{Ca}^{19} \mathrm{~F}^{+}$, each with a scan width of $\pm 4 \mathrm{~Hz}$ around the expected cyclotron frequency were measured and their results are given in Fig. 4. The first two employed the conventional excitation scheme with $900 \mathrm{~ms}$; the others used the Ramsey-type scheme of two 100-ms excitation pulses and one 700-ms waiting period (Fig. 1). Taking only into account the statistical error, the mass excess can be determined to within a $1.03 \mathrm{keV}$ uncertainty for the two resonances with conventional excitation. The uncertainty of the mass excess obtained from two Ramsey-type measurements under identical conditions is drastically reduced to $0.25 \mathrm{keV}$. All six measurements lead to an overall statistical uncertainty of the mass excess of only $0.12 \mathrm{keV}$. After consideration of systematic uncertainties of ISOLTRAP 


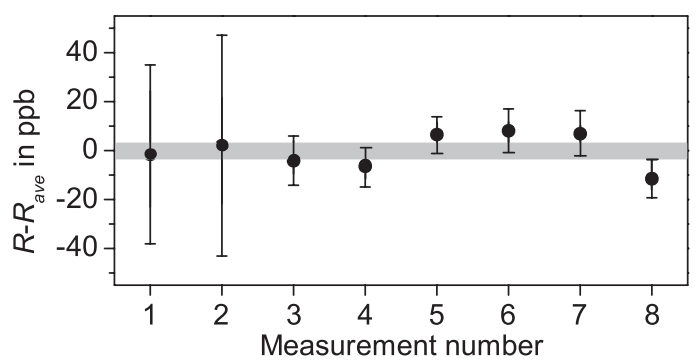

FIG. 4. Difference of the measured cyclotron-frequency ratios $R$ and their average value $R_{\text {ave }}$ between ${ }^{38} \mathrm{Ca}^{19} \mathrm{~F}^{+}$and ${ }^{39} \mathrm{~K}^{+}$. The errors are statistical. Each data point contains the same number of recorded ions. The first two points have been taken with the conventional excitation scheme, while for the last six the Ramsey method has been used.

[20] we obtain finally a frequency ratio $R$ between the ion of interest and the reference ion $\nu_{\text {ref }} / \nu_{\text {ion }}=$ $1.4622576087(34)$ (158), where the first uncertainty derives from the statistics and the second one denotes the systematic error of the measurement. Subtracting the mass excess of ${ }^{19} \mathrm{~F} M E\left({ }^{19} \mathrm{~F}\right)=-1487.39(07) \mathrm{keV}$ [21] we obtain a mass excess $M E\left({ }^{38} \mathrm{Ca}\right)=-22058.01(65) \mathrm{keV}$, which is in agreement with the previously accepted value [21] as well as with the recent value $M E\left({ }^{38} \mathrm{Ca}\right)=$ -22 058.53(28) keV [22], measured by the new Penning trap facility LEBIT at MSU and being a factor of 2 more precise. The main reason for the higher precision in the LEBIT experiment is beside better statistics that the cyclotron frequency at MSU is about a factor of 5 higher than in our experiment, since they measured doubly charged ${ }^{38} \mathrm{Ca}^{2+}$ ions in a $9.4 \mathrm{~T}$ magnetic field while we measured the singly charged molecular sideband ${ }^{38} \mathrm{Ca}^{19} \mathrm{~F}^{+}$in a $5.9 \mathrm{~T}$ magnetic field. The higher frequency leads to a smaller relative uncertainty, since the absolute uncertainty of the frequency determination is identical.

The 12 best known superallowed Fermi-type beta decays ranging from ${ }^{10} \mathrm{C}$ to ${ }^{74} \mathrm{Rb}$ yield an average $\mathrm{Ft}$ value of $\overline{F t}=$ 3072.7(0.8) s [11], which confirms the CVC hypothesis at a level of $3 \times 10^{-4}$. Further mass measurements on superallowed $\beta$ emitters for an even more stringent CVC test have been performed recently [22-24], demonstrating the continuous interest in this weak interaction and standard model test. ${ }^{38} \mathrm{Ca}$ is a candidate for testing the CVC hypothesis. Its large isospin symmetry-breaking correction of $0.73(5) \%$ [25] makes it a proper candidate for testing the validity of the calculations. Until recently the $\mathrm{Ft}$ value had a relative uncertainty of $4 \times 10^{-3}$, limited by the uncertainty of the ground-state mass, the uncertainty of the $0^{+} \rightarrow 0^{+}$branching ratio, and the accuracy of the halflife $\left(T_{1 / 2}=440(8) \mathrm{ms}\right)$, which was measured and improved at ISOLDE in parallel to our experiment. The data analysis is presently under way.

In conclusion, the method of separated oscillatory fields has been for the first time theoretically derived and applied to the mass measurement of radioactive ions confined in a
Penning trap. It allows a considerable reduction in datataking time by its improvement of precision with much less statistics. Thus, it is ideally suited for radio nuclides, especially of half-lives below $100 \mathrm{~ms}$ and production rates of only a few 100 ions/s where the uncertainty is often limited by the statistical error, as in the case of ${ }^{32} \mathrm{Ar}$ $\left(T_{1 / 2}=98 \mathrm{~ms}\right) \quad[26]$ and ${ }^{74} \mathrm{Rb}\left(T_{1 / 2}=65 \mathrm{~ms}\right)$ [27]. Further improvement by optimizing the frequency scan range and step size is presently under investigation.

This work was supported by the German BMBF under Contracts No. 06GF151 and No. 06MZ215, by the EU under Contracts No. HPMT-CT-2000-00197 (Marie Curie Fellowship) and No. RII3-CT-2004-506065 (TRAPSPEC), and by the Helmholtz association of national research centers (HGF) under Contract No. VH-NG-037. We also thank the ISOLDE Collaboration as well as the ISOLDE technical group for their assistance.

*Author to whom correspondence should be addressed. Email address: george@uni-mainz.de

[1] N. F. Ramsey, Rev. Mod. Phys. 62, 541 (1990).

[2] N. F. Ramsey, Phys. Rev. 76, 996 (1949).

[3] N. F. Ramsey, Phys. Rev. 78, 695 (1950).

[4] G. Bollen et al., Nucl. Instrum. Methods Phys. Res., Sect. B 70, 490 (1992).

[5] I. Bergström et al., Nucl. Instrum. Methods Phys. Res., Sect. A 487, 618 (2002).

[6] V. Natarajan et al., Phys. Rev. Lett. 71, 1998 (1993).

[7] G. Gräff et al., Z. Phys. A 297, 35 (1980).

[8] M. König et al., Int. J. Mass Spectrom. 142, 95 (1995).

[9] K. Blaum, Phys. Rep. 425, 1 (2006).

[10] J.C. Hardy and I. S. Towner, Phys. Rev. C 71, 055501 (2005).

[11] J. C. Hardy and I. S. Towner, Phys. Rev. Lett. 94, 092502 (2005).

[12] L. S. Brown and G. Gabrielse, Rev. Mod. Phys. 58, 233 (1986).

[13] M. Kretzschmar, in Trapped Charged Particles and Fundamental Physics, edited by Daniel H.E. Dubin and Dieter Schneider, AIP Conf. Proc. No. 457 (AIP, New York, 1999), p. 242.

[14] K. Blaum et al., Nucl. Phys. A752, 317 (2005).

[15] F. Herfurth et al., Nucl. Instrum. Methods Phys. Res., Sect. A 469, 254 (2001).

[16] G. Savard et al., Phys. Lett. A 158, 247 (1991).

[17] G. Bollen et al., Nucl. Instrum. Methods Phys. Res., Sect. A 368, 675 (1996).

[18] K. Blaum et al., J. Phys. B 36, 921 (2003).

[19] G. Bollen et al., J. Appl. Phys. 68, 4355 (1990).

[20] A. Kellerbauer et al., Eur. Phys. J. D 22, 53 (2003).

[21] G. Audi et al., Nucl. Phys. A729, 337 (2003).

[22] G. Bollen et al., Phys. Rev. Lett. 96, 152501 (2006).

[23] G. Savard et al., Phys. Rev. Lett. 95, 102501 (2005).

[24] T. Eronen et al., Phys. Rev. Lett. 97, 232501 (2006).

[25] I. S. Towner and J.C. Hardy, Phys. Rev. C 66, 035501 (2002).

[26] K. Blaum et al., Phys. Rev. Lett. 91, 260801 (2003).

[27] A. Kellerbauer et al., Phys. Rev. Lett. 93, 072502 (2004). 